\title{
Experimental evidence that a high electric field acts as an efficient external parameter during crystalline growth of bulk oxide
}

\author{
P. Hicher ${ }^{a}$, R. Haumont ${ }^{\mathrm{a}, \mathrm{b}, *}$, R. Saint-Martin ${ }^{\mathrm{a}}, \mathrm{X}$. Mininger ${ }^{\mathrm{c}}$, P. Berthet $^{\mathrm{a}}$, A. Revcolevschi ${ }^{\mathrm{a}}$ \\ a Equipe Synthèse, Propriétés et Modélisation des Matériaux, ICMMO, CNRS-UMR 8182, Université Paris Sud, 15 rue Georges Clémenceau, \\ 91405 Orsay Cedex, France \\ b Laboratoire Structures, Propriétés et Modélisation des Solides, CNRS-UMR 8580, Ecole Centrale Paris, Grande Voie des Vignes, \\ 92295 Chatenay-Malabry Cedex, France \\ ${ }^{\mathrm{c}}$ Laboratoire de Génie Electrique de Paris, SUPELEC, CNRS-UMR 8507, Université Paris Sud, 11 rue Joliot Curie, 91192 Gif-sur-Yvette, France
}

\begin{abstract}
A new crystal growth device, in which a high static external electric voltage (up to $14 \mathrm{kV}$ ) is added to a floating zone method, is described. Our first experiments show that the application of such an electric field acts like an external force, introducing a pressure effect which is in direct competition with temperature in the solid/liquid thermodynamic equilibrium. High electric fields could therefore be an additional parameter in crystal growth, opening original routes to the synthesis of new materials.
\end{abstract}

\section{Introduction}

Availability of high quality single crystals is essential for the study of intrinsic anisotropic physical properties of compounds. Crystal growth is conditioned by many parameters that involve complex thermodynamic and kinetic laws. The key to success, in crystal growth, consists in reaching an optimal thermodynamic equilibrium between the liquid phase (molten zone) and the solid phase (seed crystal). Among the various crystal growth techniques, the optical floating zone method was clearly shown in recent years to be a powerful one in a wide range of applications and for the study of both single crystal growth parameters and phase diagrams. The principle of this technique avoids using any crucible, therefore preventing possible chemical reaction between melt and crucible material. This method also allows the use of a traveling solvent procedure necessary to grow incongruently melting materials and also solid solutions with uniform compositions [1]. Besides, the technique allows also to modulate the thermal gradient at the solid-liquid interface by modifying the geometry and the nature of the heating lamps used, the growth speed and the system chemistry (composition of the feed rod, composition of

\footnotetext{
* Corresponding author at: Equipe Synthèse, Propriétés et Modélisation des Matériaux, ICMMO, CNRS-UMR 8182, Université Paris Sud, 15 rue Georges Clémenceau, 91405 Orsay Cedex, France

E-mail address: raphael.haumont@u-psud.fr (R. Haumont).
}

the solvent, when used, and gas flow); it is also possible in such a technique to modulate the partial pressure of the gas surrounding the molten zone. Previous studies have reported that this last parameter is able to convert incongruent-melting into congruentmelting [1,2]. The Gibbs free energy of the system can be consequently written with an additional term $P V$, in the form:

$G=U-T S+P V$.

In other words, a congruent-melting state is observed if the chemical potential of the liquid phase becomes equal to the solid one; thus, application of gas pressure moves boundaries in solidliquid phase diagrams by competing with thermal energy [3].

Interestingly, an electric field can also act on thermodynamic laws and on the solid/liquid interface during a growth process. Earlier studies have also shown that it is possible to modulate the morphology [4,5] and the microstructure [6,7] of a crystal by applying an electric current or an external electric field during growth. They both can affect the nucleation process [8-11], but also the partitioning of ions inside the molten zone during growth $[4,12,13]$. On one hand, crystallization under an electric current has been widely investigated but mostly on conductive materials showing that besides the change in chemical potentials induced by the electric current, several other phenomena occur during the growth process [10]. Butler and Volmer [14] showed that charge transfer phenomenon inducing nucleation can be ruled by the overvoltage. Moreover, it has been shown that the flow of an electric current can substantially alter the supercooling of the melt 
by Peltier thermoelectric cooling [15] or, at the contrary, by Joule heating [15-17], therefore modifying the driving force of the crystal growth. Finally, Lorenz force $[16,17]$ caused by an electric current pulse or electromigration [15] induced by a current flow both promote mass transport which can affect the crystal growth's rate and its quality. On the other hand, growth under an external electric potential leads to the addition of a free energy term to the Gibbs free energy [9] whose influence is bound to the dielectric permittivity of the involved medium:

$G=U-T S+P V+\S \varepsilon E^{2} V$

where $\varepsilon$ is the dielectric permittivity, $E$ the electric field strength and $V$ the volume.

Several studies, done at the microliter or milliliter level (by monitoring growth under a microscope), have shown that an external electric field can noticeably modify the phase diagram of a material by disturbing the equilibrium between the chemical potentials of solid and liquid phases [18-20]. In a system made of a high-temperature conductive oxide melt, Uda et al. have shown that most of the applied external electric potential is consumed in the electric double layer (EDL) which is presumably generated at the interface between the melt surface and the atmosphere, or at the boundary between melt and crystal embryos [20]. A huge electric field is expected to form at these boundaries: the authors have demonstrated that an electric field as high as $10^{4}-10^{5} \mathrm{~V} / \mathrm{cm}$ can be generated at the EDL during crystal growth of langasite $\left(\mathrm{La}_{3} \mathrm{Ga}_{5} \mathrm{SiO}_{14}\right)$ when applying an external electric field of $600 \mathrm{~V} / \mathrm{cm}$ [20]. As an example, such a high field could convert an incongruent-melting state into a congruent-melting one. Indeed, by developing the chemical potential expression $\eta_{L}^{j}$ (for the species $j$ in the $L$ phase), they deduced the general expression:

$\eta_{L}^{j}=\eta_{0_{L}}^{j}+k T \ln \left(\gamma_{L}^{j} C_{L}^{j}\right)+z_{L}^{j} e V+\frac{\partial}{\partial C_{L}^{j}}\left[\frac{1}{2}\left(\varepsilon_{L} E^{2}+m_{L} H^{2}+\frac{1}{c_{L}} \sigma^{2}+\ldots\right)\right]$

where, for the $j$ th species in the liquid phase, $\gamma_{L}^{j}$ is the activity coefficient, $C_{L}^{j}$ its concentration, $z_{L}^{j}$ the valence of the ion, $e$ the elementary charge, $V$ the local potential and $\varepsilon_{L}, m_{L}$ and $c_{L}$ are respectively the dielectric permittivity, the magnetic permeability and the elastic compliance of the liquid phase.

A similar expression can be derived for the solid state by changing $L$ for $S$. Eqs. (2) and (3) highlight the fact that a gas pressure $(P, \sigma)$, an electric field $(E, V)$ but also a magnetic field $H$, compete with temperature $T$ in the equilibrium occurring during crystal growth. Since dielectric permittivity $\varepsilon_{i}$, magnetic permeability $m_{i}$ and elastic compliance $c_{i}(i=S$ or $L)$ are different in the liquid and solid phases, we understand how, during crystal growth, external parameters such as $E, H$ and $\sigma$ can act on the chemical potentials $\eta_{S}$ and $\eta_{L}$; consequently shifting equilibrium between phases towards higher or lower temperatures, altering the fractions of solid and liquid in the liquid phase, and finally converting incongruent into congruent melting behavior of the material.

In this paper we report the development of a prototype device for floating-zone growth under an intense static electric field. The device enables realization of a high electrical tension, up to the breakdown voltage, between two electrodes spaced by $2.5 \mathrm{~cm}$. To our knowledge, this is the first time that a crystal growth device involving an external electric field has been associated with a mirror furnace. Besides, this set-up allows, for the first time, to study the impact of high electric field on a centimeter-size liquidsolid interface, and test previous predictions (see Eq. (3)), and micro-scale observation [18]. This development is an additional example of the great progress made since the development of the first vertical floating zone furnace in the early fifties [21].

\section{Experimental details}

We tested our new device on the growth of crystals of $\mathrm{BaCO}_{2} \mathrm{~V}_{2} \mathrm{O}_{8}$, a compound displaying a relatively low and congruent melting temperature $\left(1020^{\circ} \mathrm{C}\right)$. Lejay et al. have already reported growth of such a compound in an ordinary image furnace [22].

$\mathrm{BaCo}_{2} \mathrm{~V}_{2} \mathrm{O}_{8}$ was prepared by solid-state reaction: reagent powders of $\mathrm{BaCO}_{3}, \mathrm{Co}_{3} \mathrm{O}_{4}$ and $\mathrm{V}_{2} \mathrm{O}_{5}$ were weighed and mixed in stoichiometric proportions. The mixed and ground powders were pressed (2500 bars) and placed in an alumina boat and heated at $900{ }^{\circ} \mathrm{C}$ for $72 \mathrm{~h}$. Phase purity was checked by X-ray diffraction. The obtained samples were then crushed and shaped into cylindrical rods of $5 \mathrm{~mm}$ diameter. The rods were sintered at $900{ }^{\circ} \mathrm{C}$ during $24 \mathrm{~h}$. Crystal growth was conducted under air at a growth rate of $3 \mathrm{~mm} / \mathrm{h}$ with a counter-rotation rate of $20 \mathrm{rpm}$. Electric field was applied through a high voltage generator (Spellman SL10, $10 \mathrm{~mW}$ ).

The external electric field device used was developed to be compatible with the geometry of an NEC SC-N15 HD optical furnace (Fig. 1a) equipped with two lamps of $1500 \mathrm{~W}$ nominal power each, a sealed quartz enclosure allowing gas pressure up to 10 bars and a camera monitoring to follow progress of the growth. It is based on a lower alumina support and two pierced alumina shafts attached to the support with cement. The metallic wires used to carry the voltage are placed along the inside of the alumina shafts to protect them from the heat and to avoid any

a

b

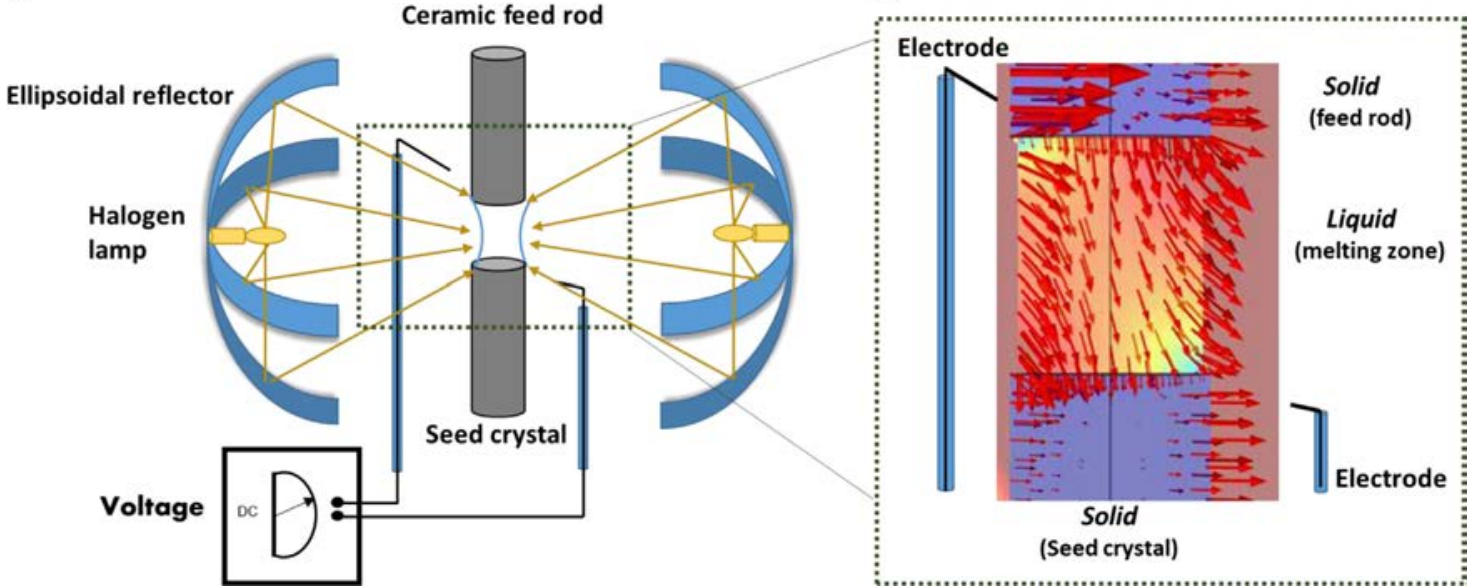

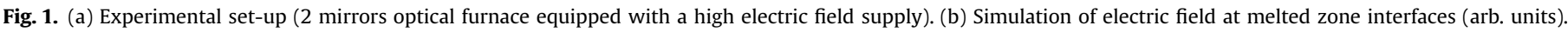


leakage current. Tips of the wires are brought both sides near the fusion zone (about $1 \mathrm{~cm}$ on both sides). Connections with the generator are made at the other end of the wires outside the mirrors' block. In order to confirm that the electric field was indeed applied through the liquid-solid interfaces during growth, simulations were carried out, taking into account the geometry used \{electrode-solid-liquid interface-molten zone-liquid-solid interface-electrode\}. Simulations were carried out with a Comsol Multiphysics software assuming steady-state conditions. Comsol is a finite element software processing numerical calculations by solving differential equations with boundary conditions. We conducted simulations in electrostatic conditions. The 3D geometry used to simulate the conditions inside the mirror furnace is a series of cylinders with different dielectric permittivities, modeling the feed rod-molten zone-crystal set-up. Results show that for an applied voltage of $10 \mathrm{kV}$, the electric field strength in the liquid zone varies from 300 to $700 \mathrm{~V} / \mathrm{cm}$ (Fig. 1b). However, one must note that due to the intrinsic anisotropy of the device, the electric field strength at the crystallization interface is even more heterogeneous, with field values varying from 20 to $700 \mathrm{~V} / \mathrm{cm}$. This last point should be improved in future experiments. Nevertheless, the present set-up allowed us already to observe remarkable effects of field application on the solid-liquid equilibrium, presented below. The observed effects were then compared to those seen under pressure and both were analyzed in terms of temperature dependence.

\section{Results}

The first effect observed when applying the electric field during crystal growth was a partial solidification of the molten zone (Fig. 2). After having stabilized the growth parameters (i.e. achieving a constant temperature through applying a constant lamps power $P=1185 \mathrm{~W}$, and a constant growth speed) and after growing $2 \mathrm{~cm}$ of material, we applied an increasing DC bias. At zero-field conditions, two distinct regions $S$ (solid) and $L$ (liquid) are separated by a clearly defined and plane interface (Fig. 2a, solid line). When increasing the electric tension, the solidification appeared more significant and the situation becomes more complex with the appearance of liquid and solid coexisting regions $(L+S)$. At constant power of lamps (i.e. constant temperature), we observe the route $L \leftrightarrow(L+S) \leftrightarrow S$, with an increasing amount of solid in the molten zone: upon increasing the applied voltage from 0 to $12 \mathrm{kV}$ at constant lamp power $(1185 \mathrm{~W})$ the volume of the liquid zone decreases gradually, showing that an external electric field has a real impact on the molten zone (Fig. $2(\mathrm{a}) \rightarrow(\mathrm{b}) \rightarrow(\mathrm{c}) \rightarrow$ (d)). By analyzing the camera pictures taken (selection and calculation of area through a commercial picture software), we have plotted $L /(S+L)$ versus the voltage applied (Fig. 3): for each applied voltage, volume fractions of liquid and solid were calculated from the 2D pictures assuming a cylindrical shape for the feed rood and the liquid zone and ratio between solid and liquid was established. To confirm the accuracy of this method based on picture analysis, experimental ratio between solid and liquid under $9.5 \mathrm{kV}$ voltage was established and compared to that obtained by analyzing the corresponding picture. To do so, picture of the material was taken under $9.5 \mathrm{kV}$ voltage just before quenching the material. The two different parts $L$ and $(L+S)$ of the quenched material were isolated and weighed to establish the ratio between solid and liquid (open triangle on Fig. 3). This ratio was then compared to the one obtained by analysis of the taken picture leading to a good agreement between the two (less than $5 \%$ error). Therefore, the same method was employed to evaluate the effect of pressure and lamps power on the $L \leftrightarrow S$ equilibrium: crystal growth was performed at constant temperature and

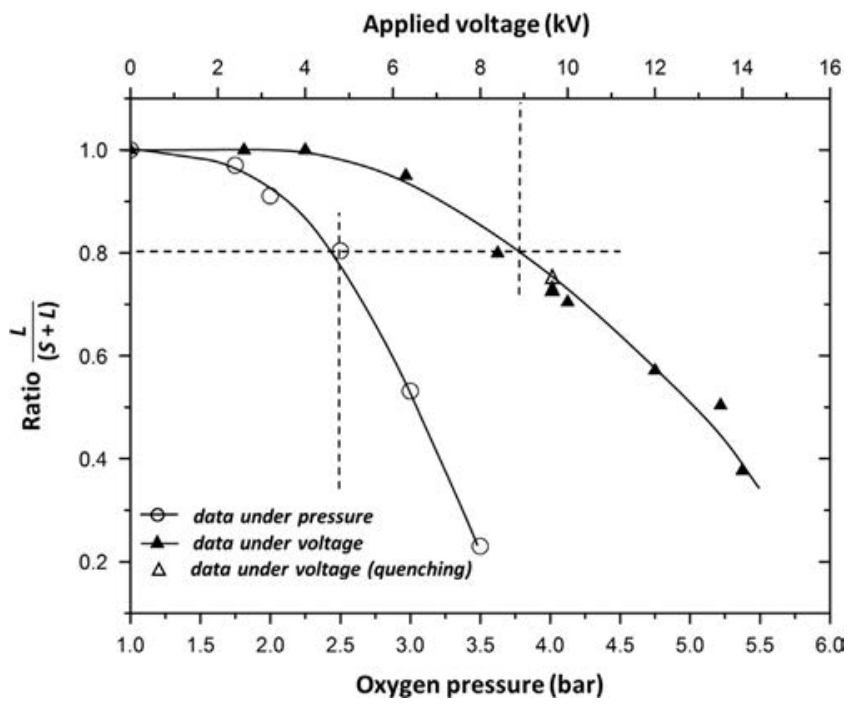

Fig. 3. Evolution of the melting zone, through the ratio $L /(S+L)$, as a function of the applied voltage and oxygen pressure $\left(\mathrm{BaCO}_{2} \mathrm{~V}_{2} \mathrm{O}_{8}\right)$. Liquid $(L)$ and solid $(S)$ parts were determined by analyzing the camera pictures of the melting zone (see Fig. 2).

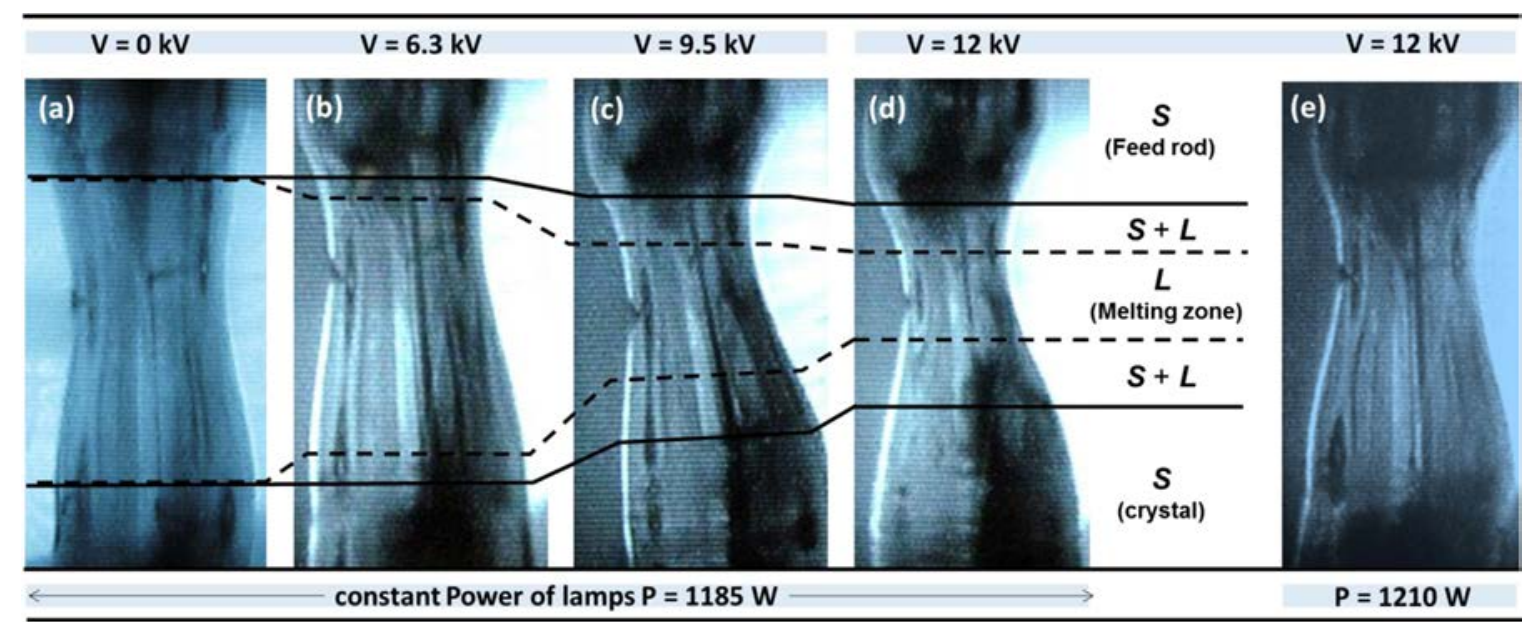

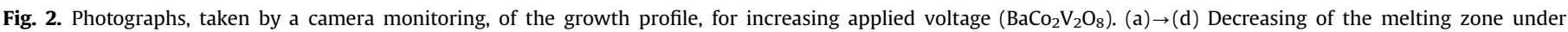

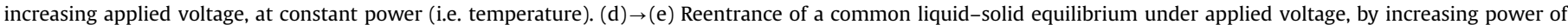
lamp (i.e. temperature). 
increasing pressure; by analyzing the taken pictures, we have plotted $L /(S+L)$ versus the pressure which was applied. Finally, the two external parameters electric tension and gas pressure can be compared in term of stability of liquid-solid equilibrium.

Results show that below about $4 \mathrm{kV}$, almost no reduction of the molten zone size occurs. However, for higher values of the electric tension, there is a spectacular reduction of the molten zone. We believe that this critical value of the field is directly dependent on the material considered (i.e. its $\varepsilon_{L}$ and $\varepsilon_{S}$, and the difference between them). Below this threshold value, the electric field contribution term $\left(\partial / \partial C_{S, L}^{j}\right)\left((1 / 2) \varepsilon_{S, L}^{i} E^{2}\right)$ to the chemical potential (Eq. (3)) can be neglected in comparison to the other.

This phenomenon is reversible: if the DC bias is switched off, the melting zone gets back to its original shape. Likewise, as we can see on pictures (d) and (e) of Fig. 2, when a high field is applied and when the molten zone size is reduced, the temperature needs to be increased to return to a common equilibrium $(L \leftrightarrow S)$. In the case of $\mathrm{BaCO}_{2} \mathrm{~V}_{2} \mathrm{O}_{8}$, it is sufficient to increase the power of the lamps from 1185 to $1210 \mathrm{~W}$ in order to obtain the volume of the initial molten zone (a). This phenomenon is analogous to the effect of pressure on solid-liquid phase equilibrium. Indeed, by increasing gas pressure on our material and using the image treatment previously described, we were able to establish a correlation between the amount of liquid in the molten zone and the applied pressure (Fig. 3). Our results show that under 3 bars of oxygen pressure, only $55 \%$ of the initial molten zone remains. Interestingly, a correlation of the results obtained under external electric voltage and under gas pressure can be done. As an example, at a constant value of the lamps power (i.e. temperature), an electric tension of $\sim 8.5 \mathrm{kV}$ has an effect similar to that of $\sim 2.5$ bars of gas pressure, by lowering the liquid ratio in the molten zone to around 0.8 (see eyes guidelines in Fig. 3). These results prove the competition between the thermal energy terms and the electric field terms in the expression of the chemical potential suggested by Uda et al. [15] in Eq. (3). Therefore, the same way gas pressure is considered important in crystal growth from the melt, application of an external electric field should be considered as an instrumental growth parameter.

Besides, Energy Dispersive X-ray (EDX, SEM) spectroscopy experiments were carried out on the quenched material. Whatever regions were analyzed $(L, L+S, S$ (crystal)), pure and single phase $\mathrm{BaCO}_{2} \mathrm{~V}_{2} \mathrm{O}_{8}$ was always observed, indicating that no decomposition due to electric field should be considered to account for the occurrence of the solid fraction in the molten zone. In the light of these observations and also in agreement with the equivalency between $E$ and $T$ described above, neither an incongruency nor a possible electrochemical decomposition of the material could be retained to explain the formation of solid part embedded in the liquid, since solid and liquid have the same chemical composition. We assume that the observed solid formation phenomenon is due to some 'cooling effect' caused by the electric field. In other words, it appears that the melting temperature $T_{m}$ increases under application of an electric field. These results are consistent with previous observations by Uda et al. [19] and Simura et al. [18] who showed that the thermodynamic equilibrium between two phases which have different dielectric permitivities can be perturbed by an electric field, leading to a modification of the melting temperature. Based on Koohpayeh's work on the modeling of temperature at different lamps power [23], we have estimated that the application of a $12 \mathrm{kV}$ voltage or of 2 bars of oxygen pressure correspond to a temperature reduction of about $25^{\circ} \mathrm{C}$. Establishing the dependence of $T_{m}$ on $E$ is a future experimental challenge which will help understanding the underlying physics.

\section{Conclusion}

In conclusion, a new mirror furnace crystal growth device using a static external electric field has been designed. A significant modification of the melting temperature and a strong change in the separation between liquid and solid states during growth have been evidenced upon application of an electric field. This study seems to reveal the existence of a critical field of about $4 \mathrm{kV}$, for the compound studied here, a value below which the electric field does not alter the melting temperature. And it indeed highlights the fact that, above this value, electric field is a pertinent parameter, which competes with temperature. In that sense, and considering in addition that an electric field can also modify nucleation, and also probably crystalline orientation during growth [24] and even partitioning of ions, we think that crystal growth under electric field is a new way to access new bulk materials, in particular oxides.

\section{References}

[1] A. Revcolevschi, J. Jegoudez, Prog. Mater. Sci. 42 (1991) 321-339.

[2] M. Hatnean, L. Pinsard-Gaudart, M. Fernandez-Diaz, S. Petit, A. Dixit, G. Lawes, R. Suryanarayanan, Eur. Phys. J. 213 (2012) 69-76.

[3] N. Wizent, L. Schramm, G. Behr, W. Löser, W. Gruner, A. Voß, B. Büchner, L. Schultz, J. Solid State Chem. 182 (2009) 2036-2040.

[4] R.A. Silva, F.M. Costa, R.F. Silva, J.P. Andreeta, A.C. Hernandes, J. Cryst. Growth 310 (2008) 3568-3572.

[5] Ongi Englander, D. Christensen, J. Kim, L. Lin, S.J.S. Morris, Nano Lett. 5 (4) (2005) 705-708.

[6] T. Bryskiewicz, J. Cryst. Growth 82 (1987) 279-288.

[7] J. Fang, P.M. Leufke, R. Kruk, D. Wang, T. Scherer, H. Hahn, Nano Today 5 (2010) $175-182$.

[8] D. Kaschiev, J. Cryst. Growth 13/14 (1972) 128-130.

[9] X. Quan, G. Chen, P. Cheng, Int. J. Heat Mass Transf. 65 (2013) 308-313.

[10] H. Conrad, Mater. Sci. Eng. A 287 (2000) 205-212.

[11] R. Dhanasekaran, P. Ramasamy, J. Cryst. Growth 79 (1986) 993-996.

[12] S. Uda, T. Tsubota, J. Cryst. Growth 312 (2010) 3650-3657.

[13] A. Feisst, A. Raüber, J. Cryst. Growth 63 (1983) 337-342.

[14] E. Budevski, G. Staikov, W.J. Lorenz, Electrochim. Acta 45 (2000) 2559-2574.

[15] T. Bryskiewicz, J. Cryst. Growth 153 (1995) 19-24.

[16] J. Li, J. Ma, Y. Gao, Q. Zhai, Mater. Sci. Eng. A 490 (2008) 452-456.

[17] X. Li, F. Lu, H. Cui, X. Tang, Trans. Nonferr. Met. Soc. China 24 (2014) 192-198.

[18] R. Simura, K. Nakamura, S. Uda, J. Cryst. Growth 310 (2008) 3873-3877.

[19] S. Uda, X. Huang, S. Koh, J. Cryst. Growth 281 (2005) 481-491.

[20] X. Huang, S. Uda, S. Koh, J. Cryst. Growth 307 (2007) 432-439.

[21] P.H. Keck, Phys. Rev. 39 (1953) 1297.

[22] P. Lejay, E. Canevet, S.K. Srivastava, B. Grenier, M. Klanjsek, C. Berthier, J. Cryst. Growth 317 (2011) 128-131.

[23] S.M. Koohpayeh, D. Fort, A. Bradshaw, J.S. Abell, J. Cryst. Growth 311 (2009) $2513-2518$.

[24] Gordon von der Gönna, R. Keding, C. Rüssel, J. Non-Cryst. Solids 243 (1999) $109-115$. 